\title{
ABSORBING BOUNDARY CONDITIONS FOR A WAVE EQUATION WITH A TEMPERATURE DEPENDENT SPEED OF SOUND
}

\author{
IGOR SHEVCHENKO \\ Department of Numerical Mathematics, Technical University of Munich \\ Garching, 85748, Germany \\ Igor.Shevchenko@ma.tum.de \\ http://www-m2.ma.tum.de \\ MANFRED KALTENBACHER \\ Institute of Mechanics and Mechatronics, Vienna University of Technology \\ Vienna, 1040, Austria \\ http://www.mec.tuwien.ac.at \\ BARBARA WOHLMUTH \\ Department of Numerical Mathematics, Technical University of Munich \\ Garching, 85748, Germany \\ http://www-m2.ma.tum.de \\ Received (Day Month Year) \\ Revised (Day Month Year)
}

\begin{abstract}
In this work, new absorbing boundary conditions (ABCs) for a wave equation with a temperature dependent speed of sound are proposed. Based on the theory of pseudo-differential calculus, first and second order ABCs for the one- and the two-dimensional wave equations are derived. Both boundary conditions are local in space and time. The well-posedness of the wave equation with the developed $\mathrm{ABCs}$ is shown through the reduction of the original problem to an equivalent one for which the uniqueness and existence of the solution has already been established. Although the second order $\mathrm{ABC}$ is more accurate, the numerical realization is more challenging. Here we use a Lagrange multiplier approach which fits into the abstract framework of saddle point formulations and yields stable results. Numerical examples illustrating stability, accuracy and flexibility of the ABCs are given. As a test setting, we perform computations for a high-intensity focused ultrasound (HIFU) application, which is a typical thermo-acoustic multiphysics problem.
\end{abstract}

Keywords: wave equation with variable coefficients; absorbing boundary conditions; pseudodifferential calculus; thermo-acoustic problem.

\section{1. Introduction}

2 In many engineering applications, multiphysics problems on unbounded domains occur.

3 Although a lot of work has been done in recent years, the numerical simulation is still chal-

4 lenging. One possible approach is to restrict the model equations to a bounded domain and

5 to impose additional boundary conditions on the fictitious boundaries. However, the solu-

6 tion is highly sensitive to the choice of the boundary conditions. Imposing simple Dirichlet 
or Neumann conditions results in non-physical effects and spurious oscillations. Suitable boundary conditions have to be transparent for outgoing waves. This type of boundary conditions is also called absorbing boundary condition (ABC), in contrast to natural and essential boundary conditions. It is commonly recognized that ABCs play a key role for unbounded domains. Over the past thirty years, ABCs have developed into a vigorous research direction including a wide spectrum of methods. The description of these techniques at length is beyond the scope of this work, and therefore we restrict ourselves to a brief overview.

In the late 1970s, the Sommerfeld-like ABCs dominated the field. ${ }^{1}$ Due to the poor approximation, spurious reflections of the waves can be observed. The necessity to suppress these reflections resulted in a number of different ABCs till the middle of 1980s. The most well-known are the Engquist-Majda $\mathrm{ABCs}^{2}$, the Bayliss-Turkel $\mathrm{ABCs}^{3}$, the DirichletNeumann map ${ }^{9}$ and others. ${ }^{4,5,6,7,8}$ The Engquist-Majda approach is based on a factorization of the wave equation leading to perfect $\mathrm{ABCs}$ which are nonlocal in space and time. To obtain local ABCs, the theory of pseudo-differential calculus has to be combined with truncated Taylor series. Quite popular are the first and second order boundary conditions. The Bayliss-Turkel technique consists in the construction of an operator annihilating the leading terms in an asymptotic expansion of the solution in the far field zone.

Later, high-order local ABCs have been developed and used mainly for the linear wave equation. ${ }^{12,13}$ At the same time investigations on the boundary element ${ }^{14}$ and the integral formulations ${ }^{15}$ as well as the infinite element approach ${ }^{16,17,18,19}$ have been carried out. In addition, the Perfectly Matched Layer technique was developed ${ }^{20}$ and had a continuation in a series of papers. ${ }^{21,22,23,24}$ This method is based on a modification of the governing equations by means of a change of coordinates. Concluding this brief survey, we would like to refer the reader to the comprehensive review articles. ${ }^{25,26,27}$

Despite the intensive research activities in this field, most results are obtained for linear problems with constant coefficients. There are only a few papers devoted to problems with variable coefficients ${ }^{28}$, convective ${ }^{30}$ and nonlinear ${ }^{28,29,31,32,33}$ terms.

In this work, we develop local ABCs for a wave equation with a temperature dependent speed of sound. ${ }^{34,35,36}$ This wave equation plays an important role in the mathematical modeling of high-intensity focused ultrasound (HIFU) applications. In particular, the solution reflects the thermo-acoustic lensing phenomenon, which can be observed in heated media as a movement of the thermal focus in the direction of the transducer. A localized temperature elevation in an initially acoustically homogeneous media causes a change in the local refraction index that leads to an acoustically inhomogeneous tissue. The thermoacoustic lensing effect is of great importance in many medical applications and can only be observed numerically if a non-linear coupled model is used. Neglecting the movement of the thermal spot during HIFU surgeries of tumors ${ }^{48,47,46}$ can lead to wrong conclusions about temperature distributions created within sonicated biotissues. The latter may result on the one hand in overheating and thus destroying healthy tissues. On the other hand, underheating the tumor possibly results in recidivism. Another example is the temperature estimation using diagnostic ultrasound. ${ }^{49,50,51}$ The thermo-acoustic lensing effect may 
substantially distort the estimates of echo shifts what in turn causes incorrect temperature predictions.

Our investigations of $\mathrm{ABCs}$ are based on the theory of pseudo-differential operators used by Engquist and Majda. ${ }^{28}$ The key ingredients are a full factorization of the wave equation with the temperature dependent speed of sound and an asymptotic expansion. Using the work of Engquist and Majda ${ }^{28}$, which considers the standard linear wave equation, as a starting point, we obtain new ABCs for the thermo-acoustic problem.

The rest of the paper is organized as follows. In Section 2, we briefly discuss the model problem. The proposed ABCs are introduced in Section 3. In Section 4, we focus on the discretization in terms of a Lagrange multiplier. Finally in Section 5, different numerical results are presented illustrating the difference between the first and second order boundary conditions.

\section{Model problem}

Let $\Omega$ be a bounded domain, see Fig. 1, in which an acoustic wave equation and the heat equation are solved. By $\Gamma_{A}$ we denote the absorbing boundary part which can be viewed as an artificial boundary reducing an unbounded domain to the bounded one. The inner boundary part is denoted by $\Gamma_{E}$, and the associated boundary conditions model a given excitation.

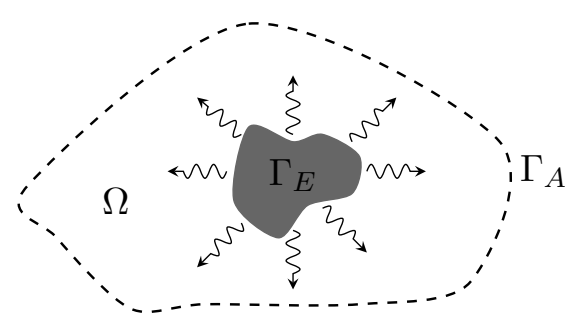

Fig. 1. General geometrical setup.

For convenience of the reader, we briefly recall how the wave equation with a temperature dependent speed of sound can be obtained. Its derivation is based on the state equation

$$
p=\rho c^{2}(T),
$$

the linearized momentum conservation equation

$$
\frac{\partial \mathbf{v}}{\partial t}=-\frac{1}{\rho_{0}} \nabla p
$$

and the linearized mass conservation

$$
\frac{\partial \rho}{\partial t}=-\rho_{0} \nabla \cdot \mathbf{v}
$$


with the Dirichlet boundary condition

$$
T(x, y, t)=T_{\mathrm{bnd}}(x, y) \quad \text { on }\left\{\Gamma_{A} \cup \Gamma_{E}\right\} \times\left(0, t_{\max }\right]
$$

$$
p=\rho_{0} \frac{\partial \psi}{\partial t} .
$$

Using this result in (4) leads to the wave equation with a temperature dependent speed of sound for the acoustic potential

$$
c^{-2}(T) \frac{\partial^{2} \psi}{\partial t^{2}}+\frac{\partial \psi}{\partial t} \frac{\partial}{\partial t} c^{-2}(T)=\Delta \psi \quad \text { in } \Omega \times\left(0, t_{\max }\right],
$$

where $t_{\max }$ is the final time at which the problem has to be solved.

To obtain a closed system for the acoustic problem, boundary and initial conditions have to be specified on $\Gamma_{E} \cup \Gamma_{A}$ and in $\Omega$, respectively. On $\Gamma_{E}$, we set the inhomogeneous Neumann boundary condition

$$
\frac{\partial \psi}{\partial n}=g(t) \quad \text { on } \Gamma_{E} \times\left(0, t_{\text {max }}\right]
$$

modeling a prescribed excitation, whereas on $\Gamma_{A}$ appropriate ABCs are set. Here $n$ is the unit normal vector to the boundary $\Gamma_{E}$ pointing outward $\Omega$. The initial conditions in $\Omega$ are

$$
\psi(x, y, 0)=\psi_{0}(x, y), \frac{\partial}{\partial t} \psi(x, y, 0)=\psi_{1}(x, y) .
$$

The acoustic model is coupled with the thermal heat conduction equation which reads

$$
\rho c_{\nu} \frac{\partial T}{\partial t}=\kappa \Delta T+\langle\mathcal{Q}(\psi)\rangle \quad \text { in } \Omega \times\left(0, t_{\max }\right]
$$




$$
T(x, y, 0)=T_{0}(x, y) \text { in } \Omega .
$$

The parameter $c_{\nu}$ denotes the specific heat capacity, $\kappa$ the thermal conductivity, and $\rho$ the density. The acoustic source term $\mathcal{Q}(\psi)$ is a temporal average of the acoustic energy being absorbed and converted to heat ${ }^{37,59,58}$, namely

$$
\mathcal{Q}(\psi)=\rho_{0}\left(\nabla \frac{\partial \psi}{\partial t} \cdot \nabla \psi+\frac{\partial \psi}{\partial t} \Delta \psi\right) .
$$

Thus the system of equations is formed by the wave equation (5) and the heat conduction equation (8).

\section{Absorbing boundary conditions}

In order to obtain $\mathrm{ABCs}$ for the wave equation (5), one can use two different approaches ${ }^{28}$. The first approach is based on the frozen coefficient theory which converts the wave equation with variable coefficients to its analog with constant coefficients by "freezing" the coefficients at a given point. For instance, the two-dimensional wave equation (24) reduces to $\alpha \partial_{t}^{2} \psi+$ $\beta \partial_{t} \psi=\Delta \psi$ with constant $\alpha, \beta$. We remark that in order to derive ABCs one can follow the idea of Engquist-Majda ${ }^{2}$ and apply the Fourier transformation in the $(y, t)$-variables. This transformation leads to the term $\partial_{x}=\sqrt{\alpha(i \tau)^{2}+\beta i \tau-(i \eta)^{2}}$ which has to be properly approximated. Here, $i \tau \leftrightarrow \partial_{t}$ and $i \eta \leftrightarrow \partial_{y}$ stand for the transfer between the frequency and time domains.

Alternatively, one can use the second approach which is based on pseudo-differential operators. We follow this approach and consider in a first step the one-dimensional wave equation which, according to (5), is

$$
c^{-2}(T) \frac{\partial^{2} \psi}{\partial t^{2}}+\frac{\partial \psi}{\partial t} \frac{\partial}{\partial t} c^{-2}(T)=\frac{\partial^{2} \psi}{\partial x^{2}} \quad \text { in }[0, a] \times\left(0, t_{\max }\right],
$$

where $\mathrm{ABCs}$ are set on the left and the right boundaries of the segment $[0, a]$. We replace the terms $c^{-2}(T)$ and $\partial_{t}\left(c^{-2}(T)\right)$ in the wave equation (11) by the variable coefficients $\alpha(x, t)$ and $\beta(x, t)$, respectively. Such a replacement leads to the following equation

$$
\mathfrak{D}_{1} \psi=0, \quad \mathfrak{D}_{1}=\alpha(x, t) \frac{\partial^{2}}{\partial t^{2}}+\beta(x, t) \frac{\partial}{\partial t}-\frac{\partial^{2}}{\partial x^{2}} .
$$

We point out that both $\alpha(x, t), \beta(x, t)$, used here, and $\alpha(x, y, t), \beta(x, y, t)$, used later for the two-dimensional case, are assumed to be $C^{\infty}$ functions in space and time. Otherwise the pseudo-differential calculus is not applicable. In the case of limited smoothness one has to use the more complex para-differential strategy. ${ }^{38,39}$

Taking into account Nirenberg's factorization ${ }^{40}$ of the operator $\mathfrak{D}_{1}$ and ideas of Engquist and Majda ${ }^{28}$, we arrive at

$$
\mathfrak{D}_{1}=-\left(\frac{\partial}{\partial x}-A\left(x, t, D_{t}\right)\right)\left(\frac{\partial}{\partial x}-B\left(x, t, D_{t}\right)\right)+R .
$$


Here $D_{t}$ stands for $-i \partial_{t}$, and $R$ is a smoothing pseudo-differential operator with the Schwartz kernel $k(x, y) \in C^{\infty}$ satisfying

$$
(1+|x-y|)^{N}\left|\frac{\partial^{\xi}}{\partial x^{\xi}} \frac{\partial^{\nu}}{\partial y^{\nu}} k(x, y)\right| \leq C_{\xi, \nu, N}, \quad \forall \xi, \nu, N \in \mathbb{N}_{0} .
$$

The pseudo-differential operators $A=A\left(x, t, D_{t}\right)$ and $B=B\left(x, t, D_{t}\right)$ have symbols $a(x, t, \tau)$ and $b(x, t, \tau)$ from the space

$$
S^{1}=S^{1}\left(\mathbb{R}^{2}\right)=\left\{f(t, \tau) \in C^{\infty}\left(\mathbb{R}^{2}\right):\left|\frac{\partial^{\xi}}{\partial t^{\xi}} \frac{\partial^{\nu}}{\partial \tau^{\nu}} f(t, \tau)\right| \leq C_{\xi, \nu}(1+|\tau|)^{1-|\nu|}, \forall \xi, \nu \in \mathbb{N}_{0}\right\} .
$$

23 In order to obtain ABCs at $x=a$ from (13), one has to make use of the fact ${ }^{41}$ that

$$
\left(\frac{\partial}{\partial x}-A\left(x, t, D_{t}\right)\right)=0
$$

is an annihilating operator for outgoing waves at $\{x=a\} \times[0,+\infty)$.

Using the factorization (13), we get

$$
\alpha(x, t) \frac{\partial^{2}}{\partial t^{2}}+\beta(x, t) \frac{\partial}{\partial t}-\frac{\partial^{2}}{\partial x^{2}}=-\frac{\partial^{2}}{\partial x^{2}}+(A+B) \frac{\partial}{\partial x}+\frac{\partial B}{\partial x}-A B+R .
$$

At the symbolic level (15) can be expressed by

$$
-\alpha(x, t) \tau^{2}+\beta(x, t) i \tau=(a+b) \frac{\partial}{\partial x}+\frac{\partial b}{\partial x}-a b+R .
$$

Now, we have to define symbols $a$ and $b$ in (16). This can be done by using asymptotic expansions given by

$$
a(x, t, \tau) \sim \sum_{j \geq 0} a_{1-j}(x, t, \tau), \quad|\tau| \rightarrow \infty
$$

and

$$
b(x, t, \tau) \sim \sum_{j \geq 0} b_{1-j}(x, t, \tau), \quad|\tau| \rightarrow \infty,
$$

where $a_{1-j}(x, t, \tau)$ and $b_{1-j}(x, t, \tau)$ are homogeneous of degree $1-j$ in $\tau$.

We note that the theorem on the product of two pseudo-differential operators ${ }^{42}$, $A(x, D) \in \Psi^{m_{1}}$ and $B(x, D) \in \Psi^{m_{2}}$ with symbols $a(x, \zeta) \in S^{m_{1}}$ and $b(x, \zeta) \in S^{m_{2}}$ respectively, yields that $C(x, D)=A(x, D) B(x, D) \in \Psi^{m_{1}+m_{2}}$ has an asymptotic expansion of its symbol $c(x, \zeta) \in S^{m_{1}+m_{2}}$ given by

$$
c(x, \zeta) \sim \sum_{|\sigma| \leq N} \frac{1}{\sigma !} D_{\zeta}^{\sigma} a(x, \zeta) \partial_{x}^{\sigma} b(x, \zeta)
$$

for every nonnegative integer $N$, and the standard multi-index notation $\sigma=\left(\sigma_{1}, \sigma_{2}, \ldots, \sigma_{k}\right)$ and $|\sigma|=\sigma_{1}+\sigma_{2}+\ldots+\sigma_{k}$. 
Combining (17) and (18), we find that

$$
c(x, t, \tau) \sim \sum_{k, l, m \geq 0} \frac{(-i)^{m}}{m !} \frac{\partial^{m}}{\partial \tau^{m}} a_{1-l}(x, t, \tau) \frac{\partial^{m}}{\partial t^{m}} b_{1-k}(x, t, \tau) .
$$

138

139

Now, substitution of (17) and (19) in (16) determines recursively the coefficients $\left\{a_{j}, b_{j}\right\}_{j \leq 0}$

$$
\left\{\begin{array}{l}
a_{-j}+b_{-j}=0, \quad j \geq 0, \\
\delta_{j 0} \beta(x, t) i \tau=-\sum_{k+l+m=j+1}\left(\frac{(-i)^{m}}{m !} \frac{\partial^{m}}{\partial \tau^{m}} a_{1-l} \frac{\partial^{m}}{\partial t^{m}} b_{1-k}\right)+\partial_{x} b_{1-j}, \quad k, l, m \geq 0,
\end{array}\right.
$$

where $\delta$ is the Kronecker delta, and

$$
a_{1}=-\sqrt{\alpha(i \tau)^{2}}, \quad b_{1}=-a_{1} .
$$

For simplicity in exposition, we present additionally to $a_{1}$ and $b_{1}$ only two coefficients $a_{0}$ and $b_{0}$

$$
a_{0}=\frac{1}{2 a_{1}}\left(\beta(x, t) i \tau+\frac{\partial a_{1}}{\partial x}+i \frac{\partial a_{1}}{\partial \tau} \frac{\partial a_{1}}{\partial t}\right), \quad b_{0}=-a_{0} .
$$

The use of the asymptotic expansion (17a) in (14) and the first $k$ terms enable us to rewrite the boundary conditions in the form

$$
\left(\frac{\partial}{\partial x}-\sum_{j=0}^{k} a_{1-j}(x, t, \tau)\right) \psi=0 \quad \text { at }\{x=a\} \times[0,+\infty) .
$$

Finally, substitution of the coefficients $a_{1}$ and $a_{0}$ in the boundary condition (21) gives the first order $\mathrm{ABC}$ in the following form

$$
\left(\frac{\partial}{\partial x}+\frac{1}{c(T)} \frac{\partial}{\partial t}+\frac{1}{2 c(T)}\left(\frac{\partial}{\partial x} c(T)-\frac{1}{c(T)} \frac{\partial}{\partial t} c(T)\right)\right) \psi=0 \quad \text { at }\{x=a\} \times[0,+\infty) .
$$

The ABC for the left boundary is obtained analogously with the only difference that the sign in (14) is changed from minus to plus, namely

$$
\left(\frac{\partial}{\partial x}-\frac{1}{c(T)} \frac{\partial}{\partial t}-\frac{1}{2 c(T)}\left(\frac{\partial}{\partial x} c(T)-\frac{1}{c(T)} \frac{\partial}{\partial t} c(T)\right)\right) \psi=0 \quad \text { at }\{x=0\} \times[0,+\infty) .
$$

Remark 3.1. It is important to stress out that we define the order of the $A B C$ by the order of the principal part of the differential operator in this $A B C$.

We can now derive transparent boundary conditions for the two-dimensional case. First, we obtain $\mathrm{ABCs}$ on the wall $x=a$ and then extend the result for the whole domain $\Omega=(0, a) \times(0, b)$. The derivation starts from the replacement of the terms $c^{-2}(T)$ and 
$\partial_{t}\left(c^{-2}(T)\right)$ in the wave equation (5) with the variable coefficients $\alpha(x, y, t)$ and $\beta(x, y, t)$. Thus the wave equation becomes

$$
\mathfrak{D}_{2} \psi=0, \quad \mathfrak{D}_{2}=\alpha(x, y, t) \frac{\partial^{2}}{\partial t^{2}}+\beta(x, y, t) \frac{\partial}{\partial t}-\frac{\partial^{2}}{\partial x^{2}}-\frac{\partial^{2}}{\partial y^{2}} .
$$

As in the one dimensional case, we factorize the operator $\mathfrak{D}_{2}$ by

$$
\mathfrak{D}_{2}=-\left(\frac{\partial}{\partial x}-A\left(x, y, t, D_{y}, D_{t}\right)\right)\left(\frac{\partial}{\partial x}-B\left(x, y, t, D_{y}, D_{t}\right)\right)+R .
$$

Here $A\left(x, y, t, D_{y}, D_{t}\right)$ and $B\left(x, y, t, D_{y}, D_{t}\right)$ are pseudo-differential operators with symbols $a(x, y, t, \eta, \tau)$ and $b(x, y, t, \eta, \tau)$, respectively. These symbols can then be recursively determined from the factorization analogously to (16), namely

$$
-\alpha(x, y, t) \tau^{2}+\beta(x, y, t) i \tau+\eta^{2}=(a+b) \frac{\partial}{\partial x}+\frac{\partial b}{\partial x}-a b+R .
$$

A similar argument as in the one-dimensional case yields the coefficients

$$
\begin{aligned}
& a_{1}=-\sqrt{\eta^{2}-\alpha(x, y, t) \tau^{2}}, \quad b_{1}=-a_{1}, \\
& a_{0}=\frac{1}{2 a_{1}}\left(\beta(x, y, t) i \tau+\frac{\partial a_{1}}{\partial x}-i^{2} \frac{\partial^{2} a_{1}}{\partial \eta \partial \tau} \frac{\partial^{2} a_{1}}{\partial y \partial t}\right), \quad b_{0}=-a_{0} .
\end{aligned}
$$

So far, the derivation followed exactly the same lines as in the one-dimensional case. From now on, there is a difference. Due to the desired locality of the boundary condition, we have to approximate the square root in (27). There are several ways how to do such approximations. Some of them are based on Padé and Taylor series ${ }^{2}$, others use rational ${ }^{43}$ or least-squares approximations. ${ }^{44}$ In this work, we expand the square root in a Taylor series up to the second order of accuracy. Substitution of the coefficients $a_{1}, a_{0}$ in the two-dimensional analog of $(21)$

$$
\left(\frac{\partial}{\partial x}-\sum_{i=0}^{k} a_{1-i}(x, y, t, \eta, \tau)\right) \psi=0
$$

gives the first

$$
\left(\frac{\partial}{\partial x}+\frac{1}{c(T)} \frac{\partial}{\partial t}+\frac{1}{c(T)}\left(\frac{1}{2} \frac{\partial}{\partial x} c(T)-\frac{1}{c(T)} \frac{\partial}{\partial t} c(T)\right)\right) \psi=0
$$

and the second

$$
\left(\frac{1}{c(T)} \frac{\partial^{2}}{\partial x \partial t}+\frac{1}{c^{2}(T)} \frac{\partial^{2}}{\partial t^{2}}-\frac{1}{2} \frac{\partial^{2}}{\partial y^{2}}+\frac{1}{c^{2}(T)}\left(\frac{1}{2} \frac{\partial}{\partial x} c(T)-\frac{1}{c(T)} \frac{\partial}{\partial t} c(T)\right) \frac{\partial}{\partial t}\right) \psi=0
$$

order ABCs on the wall $x=a$. The boundary conditions on the walls $x=0, y=0$ and $y=b$ can be derived in the same way. 
Introducing the normal $n$ and the tangential $\tau$ derivatives, the ABCs on the entire absorbing boundary $\Gamma_{A}$ of the domain $\Omega$ can be written as

$$
\left(\frac{\partial}{\partial n}-\frac{1}{c(T)} \frac{\partial}{\partial t}+\frac{1}{c(T)}\left(\frac{1}{2} \frac{\partial}{\partial n} c(T)-\frac{1}{c(T)} \frac{\partial}{\partial t} c(T)\right)\right) \psi=0
$$

and

$$
\left(\frac{1}{c(T)} \frac{\partial^{2}}{\partial n \partial t}-\frac{1}{c^{2}(T)} \frac{\partial^{2}}{\partial t^{2}}+\frac{1}{2} \frac{\partial^{2}}{\partial \tau^{2}}-\frac{1}{c^{2}(T)}\left(\frac{1}{2} \frac{\partial}{\partial n} c(T)-\frac{1}{c(T)} \frac{\partial}{\partial t} c(T)\right) \frac{\partial}{\partial t}\right) \psi=0 .(30
$$

It is worth to point out that for the one-dimensional case the ABCs can only be improved if additional terms in the asymptotic expansion of the symbol $a(x, t, \tau)$ are taken into account. However, in the two-dimensional setting, also higher-order approximations of the square root in (27) result in more accurate boundary conditions.

Remark 3.2. If the constant speed of sound is used in the boundary conditions (30), one arrives at the first order

$$
\left(\frac{\partial}{\partial n}-\frac{1}{c} \frac{\partial}{\partial t}\right) \psi=0
$$

and the second order

$$
\left(\frac{1}{c} \frac{\partial^{2}}{\partial n \partial t}-\frac{1}{c^{2}} \frac{\partial^{2}}{\partial t^{2}}+\frac{1}{2} \frac{\partial^{2}}{\partial \tau^{2}}\right) \psi=0
$$

\section{Engquist-Majda $A B C s$ on $\Gamma_{A}$.}

Thus the boundary conditions (30) can be regarded as a natural extension of the EngquistMajda ABCs (31) to a wave equation with temperature dependent speed of sound.

The obtained boundary conditions (30) give rise to the question: Is the wave equation with the new ABCs well-posed? In order to show that the initial boundary value problem $(5),(7),(30)$ is well-posed one has to prove the uniqueness and the existence of the solution for the original problem or to rewrite it as an equivalent problem for which the well-posedness is already established. For instance, the well-posedness of the initial boundary value problem (5),(7),(30) with constant speed of sound has been completely analyzed in a half-space ${ }^{28,53}$ and for a corner problem. ${ }^{52}$ Thus the only step we have to perform is to reduce our problem to the one with constant speed of sound. Such a reduction can be based on the Gordienko technique ${ }^{54}$ which consists of three main steps: (i) "Freeze" the coefficients and extract the principal part of the differential operator in the wave equation (5) and in the boundary condition (30); (ii) Check that the obtained system satisfies the uniform Lopatinskii condition; (iii) Reduce the problem to a symmetric hyperbolic system and prove the dissipativity of the boundary condition.

In our situation, we do not have to work out all three steps. It is sufficient to apply only the first step which already leads to the standard wave equation with constant speed of sound and the Engquist-Majda ABCs (31) for which the well-posedness results are wellknown. 


\section{Discretization}

In this section, we apply a standard low order finite element method for the two-dimensional thermo-acoustic problem (5)-(10) with ABCs (30). The weak formulation of the wave equation (5) reads as

$$
\int_{\Omega} \frac{1}{c^{2}} \frac{\partial^{2} \psi}{\partial t^{2}} \phi d \Omega+\int_{\Omega}(\nabla \psi \cdot \nabla \phi) d \Omega-\int_{\Gamma_{A}} \frac{\partial \psi}{\partial n} \phi d \Gamma_{A}=\int_{\Gamma_{E}} g \phi d \Gamma_{E}
$$

for all suitable test functions $\phi$.

The use of ABC (30a) in (32) is obvious. However, the situation is different in case of the second order condition (30b). A straightforward substitution of (30b) in the boundary integral along $\Gamma_{A}$ is not possible due to the lack of the term $\partial_{n} \psi$. Thus, we use a Lagrange multiplier based approach which consists of the following steps. ${ }^{56}$

Firstly, a Lagrange multiplier $\Lambda$ on the absorbing boundary $\Gamma_{A}$ is introduced and the term $-\partial_{n} \psi$ is replaced by $\Lambda$ in (32). For the Lagrange multiplier we can use any stable approach well-known from the mortar setting. We point out that each discontinuity of the normal on $\Gamma_{A}$ is handled as a crosspoint within the mortar context.

Secondly, we restate the boundary condition (30b) weakly in terms of $\Lambda$

$$
\int_{\Gamma_{A}}\left(-\frac{1}{c} \frac{\partial \Lambda}{\partial t}-\frac{1}{c^{2}} \frac{\partial^{2} \psi}{\partial t^{2}}+\frac{1}{2} \frac{\partial^{2} \psi}{\partial \tau^{2}}-\frac{1}{c^{2}}\left(\frac{1}{2} \frac{\partial c}{\partial n}-\frac{1}{c} \frac{\partial c}{\partial t}\right) \frac{\partial \psi}{\partial t}\right) \mu d \Gamma_{A}=0,
$$

where $\mu$ is a test function. Thus, one has a subsystem of two equations for the unknowns $(\psi, \Lambda)$. Due to the temperature dependent speed of sound, this subsystem forms together with the heat conduction equation (8) a two-sided coupled problem.

The algebraic formulation of this problem can be expressed as a semidiscrete system of nonlinear ordinary differential equations

$$
\left(\begin{array}{lll}
0 & 0 & 0 \\
0 & \mathbf{M} & 0 \\
0 & \mathbf{B} & 0
\end{array}\right)\left(\begin{array}{l}
\ddot{\mathbf{T}} \\
\ddot{\mathbf{\Psi}} \\
\ddot{\mathbf{\Lambda}}
\end{array}\right)+\left(\begin{array}{ccc}
\mathbf{C} & \mathcal{Q} & 0 \\
0 & \mathbf{N} & 0 \\
0 & \mathbf{R} & \mathbf{D}
\end{array}\right)\left(\begin{array}{l}
\dot{\mathbf{T}} \\
\dot{\mathbf{\Psi}} \\
\dot{\boldsymbol{\Lambda}}
\end{array}\right)+\left(\begin{array}{ccc}
\widetilde{\mathbf{K}} & 0 & 0 \\
0 & \mathbf{K} & \mathbf{D}^{\mathrm{T}} \\
0 & \widetilde{\mathbf{C}} & 0
\end{array}\right)\left(\begin{array}{l}
\mathbf{T} \\
\mathbf{\Psi} \\
\boldsymbol{\Lambda}
\end{array}\right)=\left(\begin{array}{l}
\mathbf{0} \\
\mathbf{f} \\
\mathbf{0}
\end{array}\right)
$$

with standard notations for the mass matrix $\mathbf{M}$ and the damping matrix $\mathbf{C}$. The stiffness matrices for the heat conduction equation and the wave equation are denoted by $\mathbf{K}$ and $\widetilde{\mathbf{K}}$, respectively. The matrices $\mathbf{B}(\mathbf{T}), \mathbf{R}(\mathbf{T}), \mathbf{D}(\mathbf{T})$ and $\widetilde{\mathbf{C}}(\mathbf{T})$ are responsible for the coupling between the boundary condition (33) and the wave equation (32). In addition, the matrices $\mathcal{Q}(\boldsymbol{\Psi}, \boldsymbol{\Lambda})$ and $\mathbf{N}(\mathbf{T})$ reflect the nonlinear terms in the wave equation and the heat equation.

In order to discretize the system of equations (34) in time, the classical Newmark scheme can be applied. ${ }^{60}$ However, the wave propagation and the heat conduction are processes evolving on different time scales. For instance, the characteristic time of temperature changes lies in the range of seconds while high intensity ultrasound waves require hundredths of a microsecond to be accurately resolved. Thus, in order to accurately resolve the physical processes on different time scales we apply a multi-time stepping integration method ${ }^{56}$ 
which is more accurate and works faster compared to the conventional technique used in HIFU applications. ${ }^{48,47,46}$

\section{Numearical results}

\subsection{Model problem}

In this section, we study numerically the performance of the newly developed ABCs (30a) and (30b) for the thermo-acoustic problem (5)-(10). We will also apply the standard Engquist-Majda boundary conditions to demonstrate that a naive application of these ABCs which are tailored for the linear wave equation does not guarantee satisfying results when applying it to the wave equation with temperature dependent speed of sound.

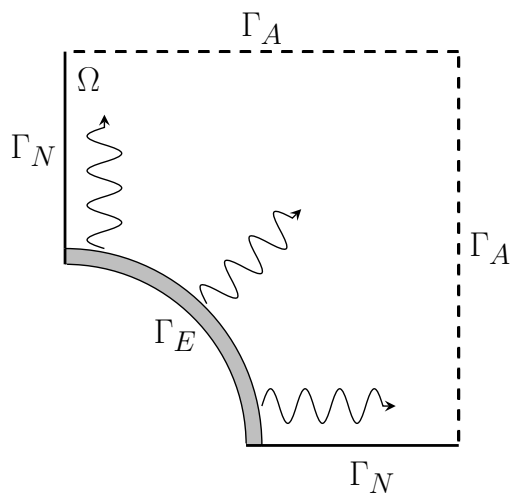

Fig. 2. Geometrical setup for the thermo-acoustic problem.

We consider cylindrical waves on a unit square as displayed in Fig. 2. The computational domain $\Omega \subset \mathbb{R}^{2}$ is filled up with water for which the constant and the temperature dependent speed of sound are assumed to be ${ }^{46} c=c_{0}$ and

$c(T)=c_{0}+5.0371 T-5.8085 \cdot 10^{-2} T^{2}+3.3420 \cdot 10^{-4} T^{3}-1.4780 \cdot 10^{-6} T^{4}+3.1464 \cdot 10^{-9} T^{5}$

where $c_{0}=1402.39$. On the boundary $\Gamma_{E}$ we prescribe the normal derivative of the acoustic potential (inhomogeneous Neumann boundary condition) to model a mono-frequency transducer vibrating at a frequency of $5 \mathrm{kHz}$. Furthermore on $\Gamma_{A}$, we set the $\mathrm{ABCs}$ and on $\Gamma_{N}$, homogeneous Neumann boundary conditions are used to guarantee symmetry. For the thermal computation, we set for the temperature a homogeneous Neumann boundary condition on $\Gamma_{E}$ and a homogeneous Dirichlet boundary condition on $\Gamma_{N} \cup \Gamma_{A}$.

In order to compare different transparent boundary conditions for the setup in Fig.2, we first compute a solution in the domain $\Omega^{\prime} \ni \Omega$ representing a square domain with the side of length $c t_{\max }$, which is than used as a reference solution when computing the $L^{\infty}$-norm relative error $\delta$ for the numerical results obtained on the restricted domain $\Omega$. Furthermore, 
13 bilinear finite elements per wavelength are used in the numerical simulations, and the time step size is set to $20 \mathrm{~ms}$, which corresponds to 10 time samples per time period.
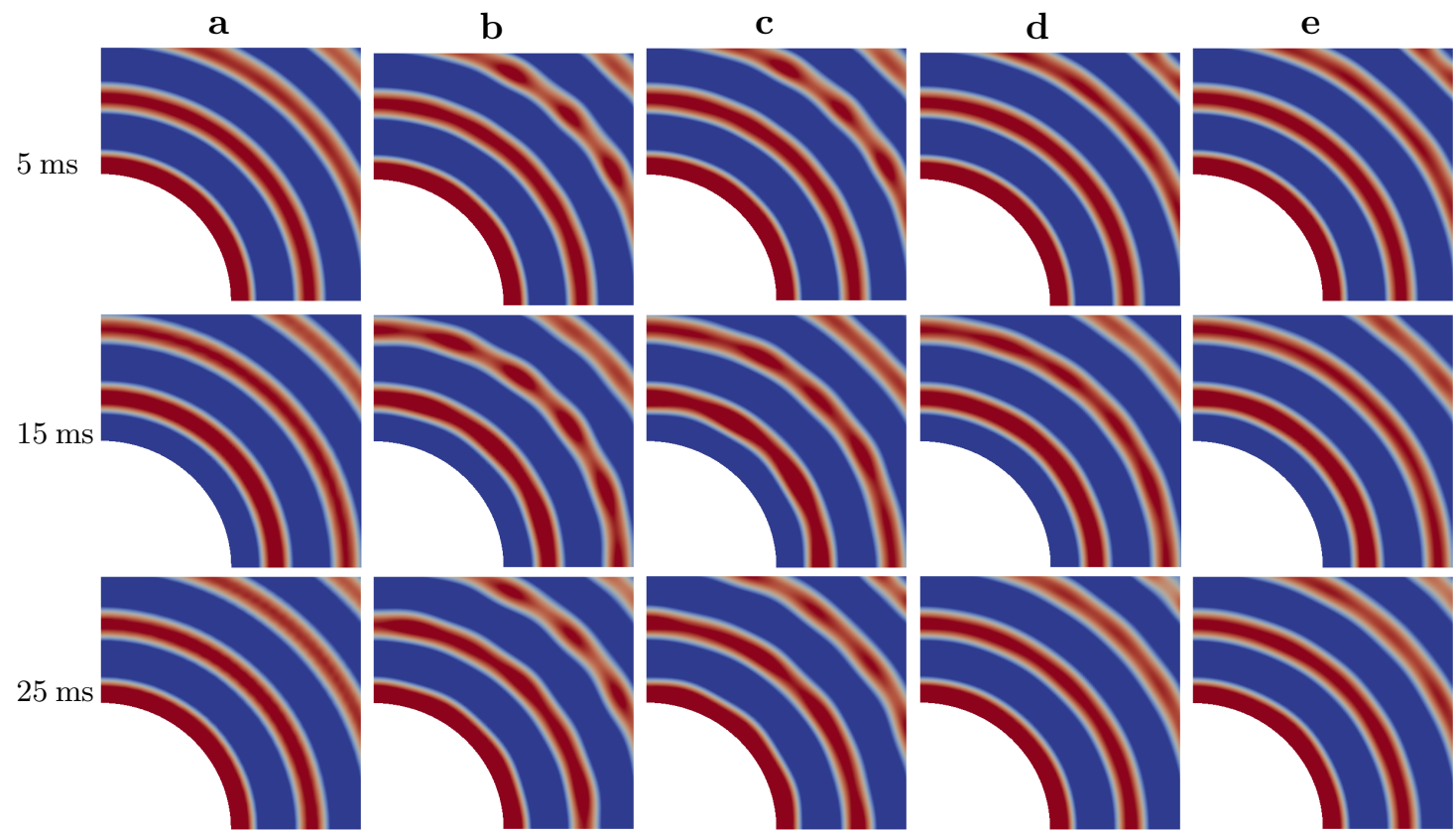

Fig. 3. The evolution of the acoustic field for the setup in Fig. 2. The row a corresponds to the reference solution, the rows $\mathbf{b}$ and $\mathbf{c}$ stand for the first and second order Engquist-Majda ABCs while the rows $\mathbf{d}$ and e represent the boundary condition (30a) and (30b).

Figure 3 displays the contour levels of the acoustic pressure at different characteristic time steps. The discrepancy between the first and second order Engquist-Majda ABCs and the proposed transparent boundary conditions (30a) and (30b) is clearly visible. This result is also reflected in Fig. 4, which shows the evolution of the relative error $\delta$ in time.

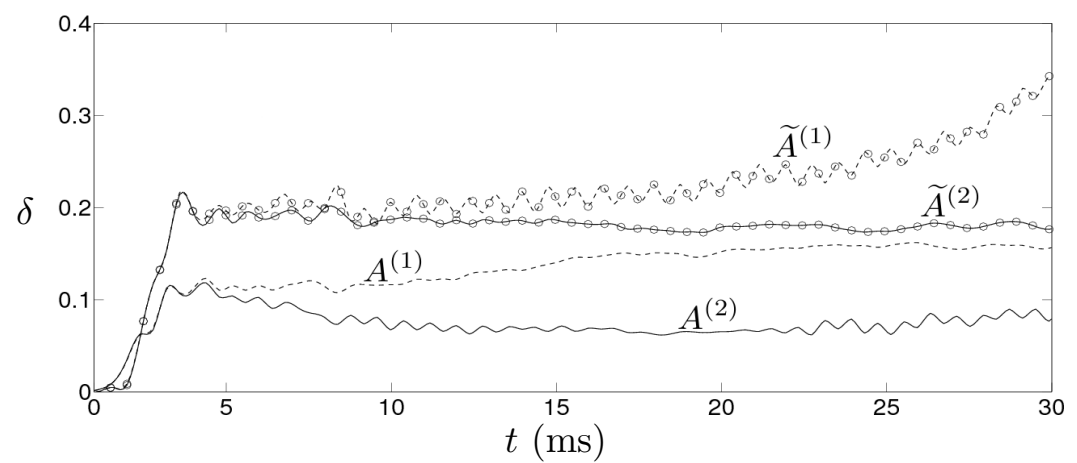

Fig. 4. $L^{\infty}$-norm relative error (vertical axis) of the temperature vs. time in milliseconds (horizontal axis) for the setup in Fig. 2. The first (30a) and second (30b) order ABCs are marked by $A^{(1)}$ and $A^{(2)}$, respectively. The first (31a) and second (31b) order Engquist-Majda ABCs are denotes by $\widetilde{A}^{(1)}$ and $\widetilde{A}^{(2)}$, respectively. 
Furthermore, Fig. 4 demonstrates the strong improvement of the second order ABCs compared to the first order ones.

\subsection{HIFU heating example}

In this section, we study how the performance of the proposed ABCs (30a) and (30b) depends on the excitation frequency. We consider a rectangular computational domain $\Omega=$ $[0,20 \mathrm{~mm}] \times[0,25 \mathrm{~mm}]$ consisting of a human liver tissue for which, in accordance to Connor and Hynynen ${ }^{46}$, the temperature dependent speed of sound is given by the followinf polynomial

$c(T)=1529.3+1.6856 T+6.1131 \cdot 10^{-2} T^{2}-2.2967 \cdot 10^{-3} T^{3}+2.2657 \cdot 10^{-5} T^{4}-7.1795 \cdot 10^{-8} T^{5}$.

This polynomial adequately describes the speed of sound within the temperature interval $\left[30^{\circ} \mathrm{C}, 90^{\circ} \mathrm{C}\right]$ which is suitable for many HIFU treatments.

On all boundaries of the computational domain, except for the bottom part, we set ABCs (30). We use a monofrequency transducer $\Gamma_{E}$, located on the bottom of the computational domain, with an aperture of $20 \mathrm{~mm}$, producing sinusoidal waves. We use frequencies $\omega=\{0.8 \mathrm{MHz}, 1.0 \mathrm{MHz}, 1.2 \mathrm{MHz}\}$ which are typical for HIFU therapy. The time step for the temperature $T$ is set to be $\Delta t=0.01 \mathrm{~s}$, and the acoustic potential $\psi$ is resolved with the time step $\delta t$ to have 20 time samples per time period for each of the frequencies $\omega$. In space, 20 finite elements per wavelength are used. For the sake of convenience in exposition, the acoustic pressure, the temperature field and the time are normalized to their maximum values, and we set $T_{\mathrm{bnd}}=37^{\circ} \mathrm{C}$.

The primary goal of this work is to analyze the efficiency and robustness of the developed ABCs (30). However, one of the most important factors which determines the success of any HIFU therapy is the knowledge of the temperature distribution created within sonicated biotissues. Thus, we also study how the imperfection of the ABCs influences the temperature field.

In a first step, we consider the lowest frequency $\omega=0.8 \mathrm{MHz}$. Fig. 5 shows the acoustic pressure and the temperature field for the first and second order ABCs (30a) and (30b) as well as for the first and second order Engquist-Majda ABCs.

As it can be seen from Fig. 5(c), the second order ABC (30b) yields in comparison to the first order condition (30a) a better numerical approximation. Even for small simulation times $(t=0.4)$, the first order ABC (30a) shows pollution of the temperature distribution appearing in the upper part of Fig. 5(d),II. For larger times, the pollution effect increases (see Fig. 5(d),II for $t=0.6$ ) and at $t=1.0$ the upper part of the temperature field is completely distorted by reflected waves. In contrast, the second order ABC (30b) gives good results throughout the entire simulation, and the pollution effect of the wave solution in the temperature field is considerably reduced (see Fig. 5(d),III) for (30b).

Let us now address the results obtained for the first and second order Engquist-Majda ABCs (31). Already from the very beginning $(t=0.4)$, the first order condition (31a) gives a lower accuracy compared to the ABC (30a). The situation becomes worse as time advances 


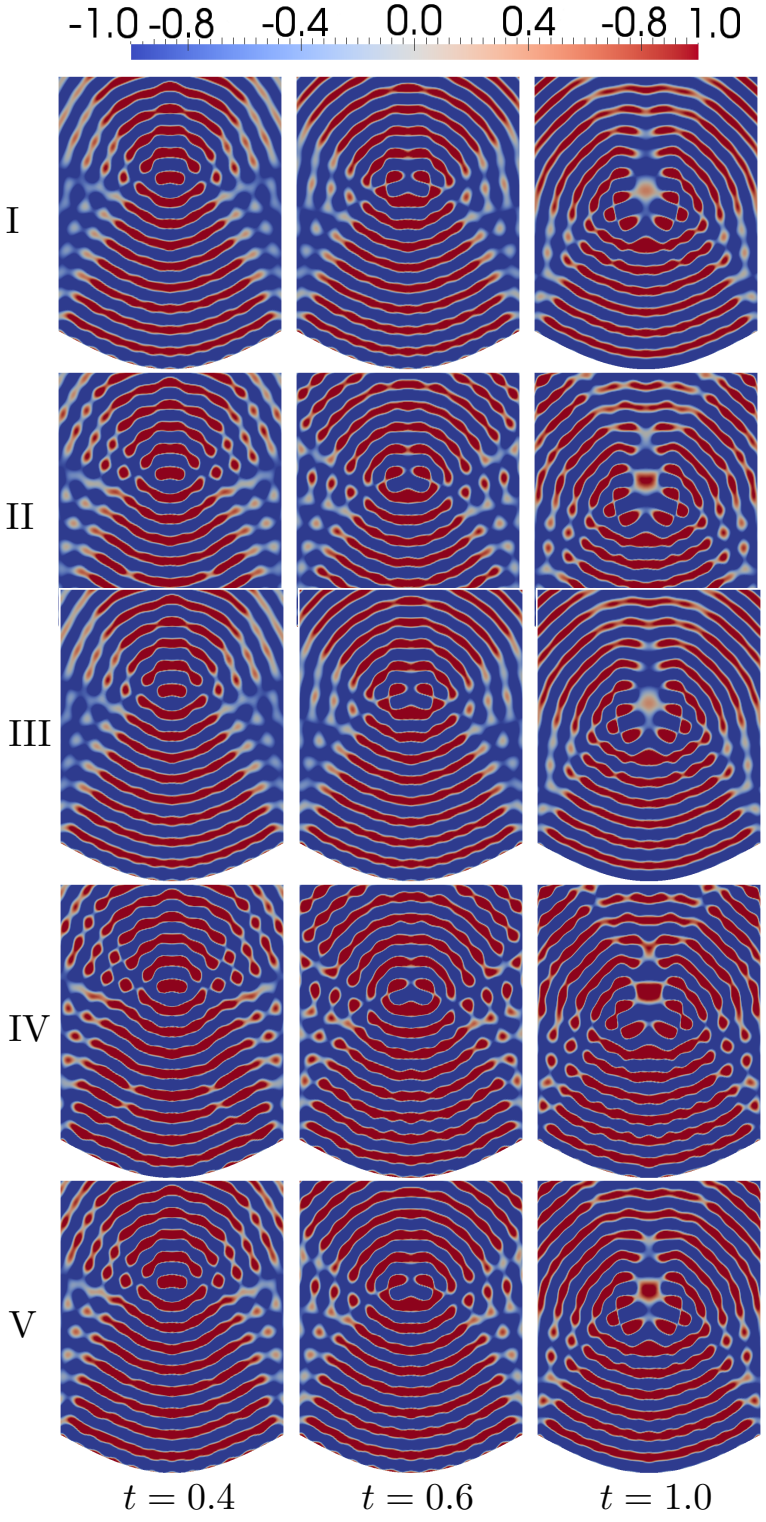

(c) Acoustic pressure.

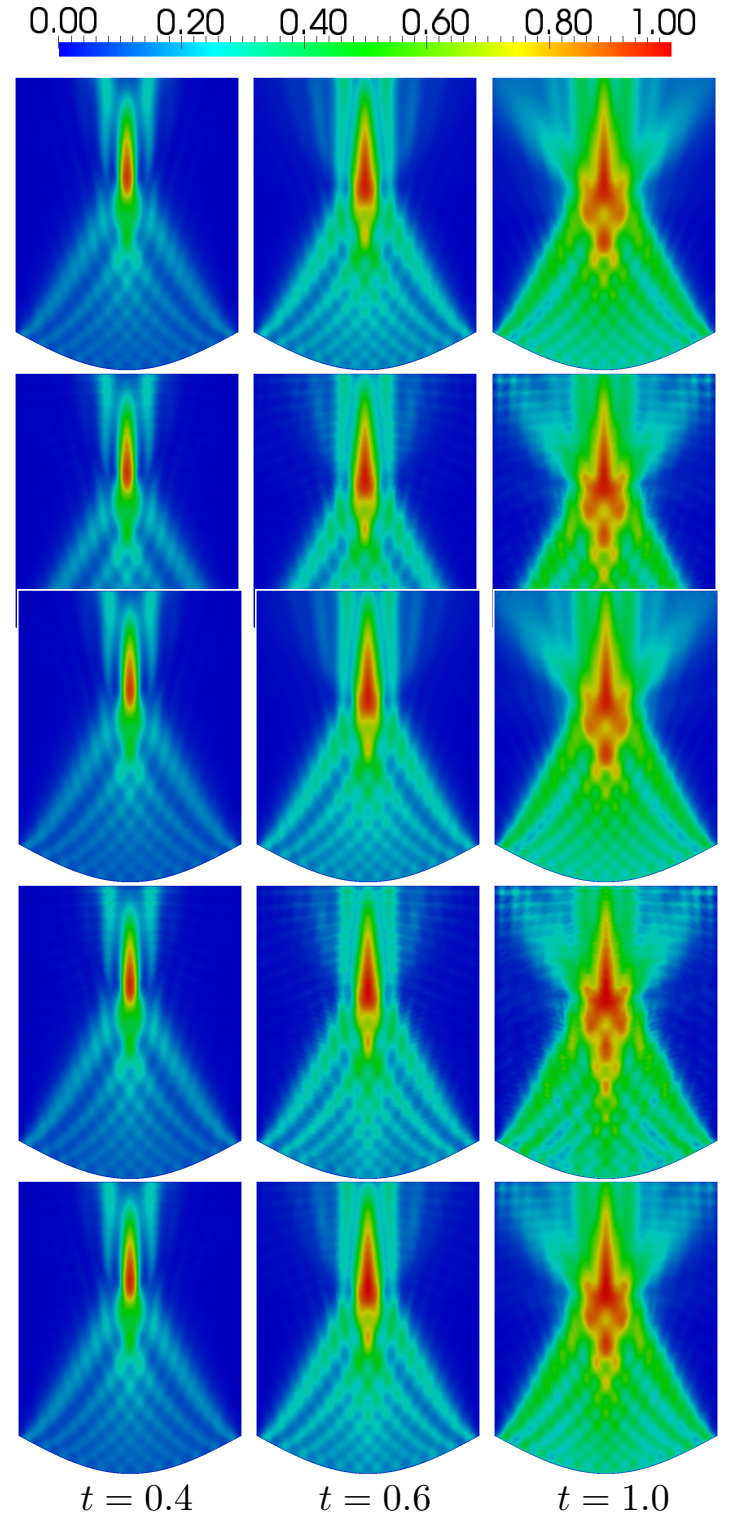

(d) Temperature field.

Fig. 5. A series of snapshots of the acoustic pressure and the temperature field for the excitation frequency $\omega=0.8 \mathrm{MHz}$ at different times. The reference solution is in row I. The first and second order ABCs (30) are in rows II and III, whereas the first and second order Engquist-Majda ABCs (31) are in rows IV and V, respectively.

(see Fig. 5,IV for $t>0.4$ ). Moreover, the use of the second order Engquist-Majda boundary condition (see Fig. 5,V) does not significantly change the situation, and the solution is still substantially polluted by reflected waves. Thus we can conclude that a naive application of $\mathrm{ABCs}$ which have been developed for the linear wave equation does not provide satisfying 


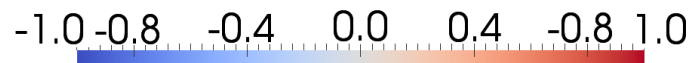

I
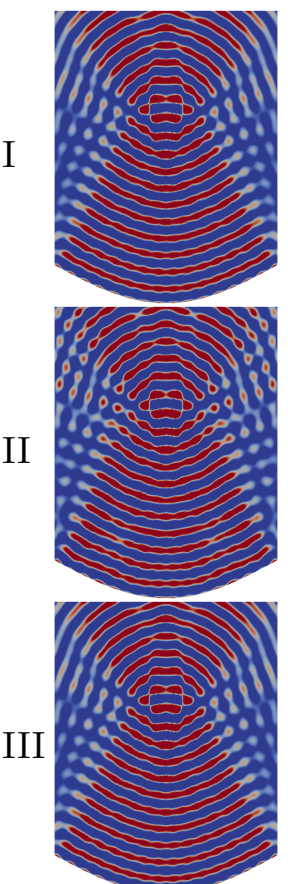

$t=0.4$
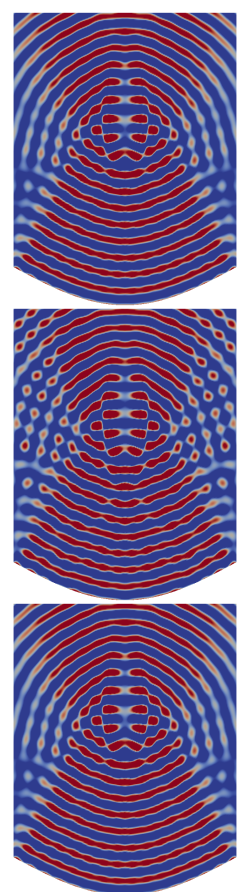

$t=0.6$
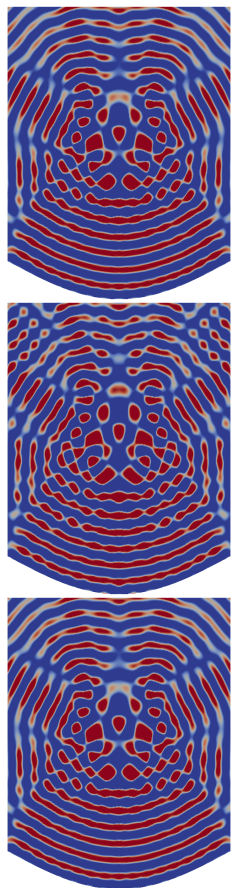

$t=1.0$

(a) Acoustic pressure
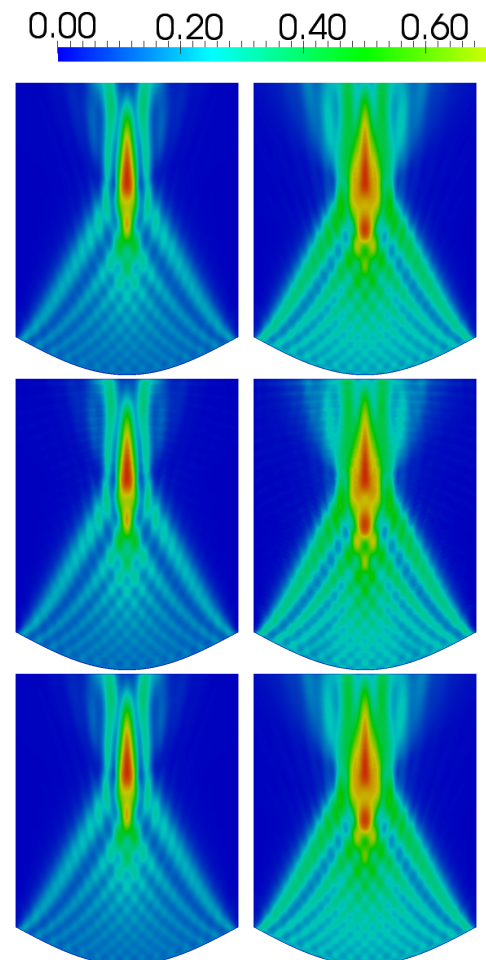

$t=0.4$

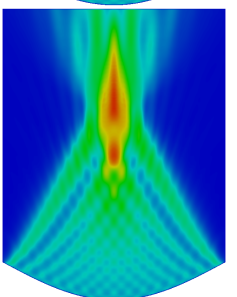

$t=0.6$

(b) Temperature field.
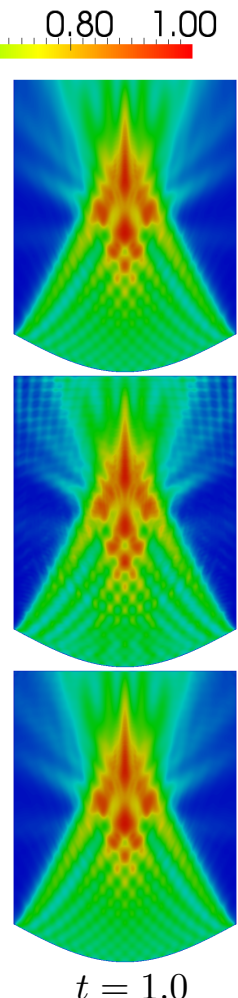

Fig. 6. A series of snapshots of the acoustic pressure and the temperature field for the excitation frequency $\omega=1.0 \mathrm{MHz}$ at different times. The reference solution is in row I. The first and second order ABCs (30) are in rows II and III, respectively.

It can be easily observed from Fig. 6 that the proposed first order $\mathrm{ABC}$ (30a) is much more sensitive to the excitation frequency than the second order ABC (30b). In comparison to Fig.5, Fig. 6 shows much higher spurious oscillations for the first order case in the wave solution, and as a consequence the temperature distribution is more distorted.

Finally, we set $\omega=1.2 \mathrm{MHz}$ and report the results in Fig. 7. Increasing the frequency from $1.0 \mathrm{MHz}$ to $1.2 \mathrm{MHz}$ leads to a quite poor numerical approximation for the acoustic pressure as well as for the temperature with the use of the first order ABC (30a). As it can be clearly seen in the second row of Fig. 7, the acoustic pressure shows a wrong pattern which superposes the global structure of the temperature distribution on a finer scale. Moreover, even the thermal spot starts to exhibit artificial details (Fig. 7(b),II for $t=1.0$ ). 


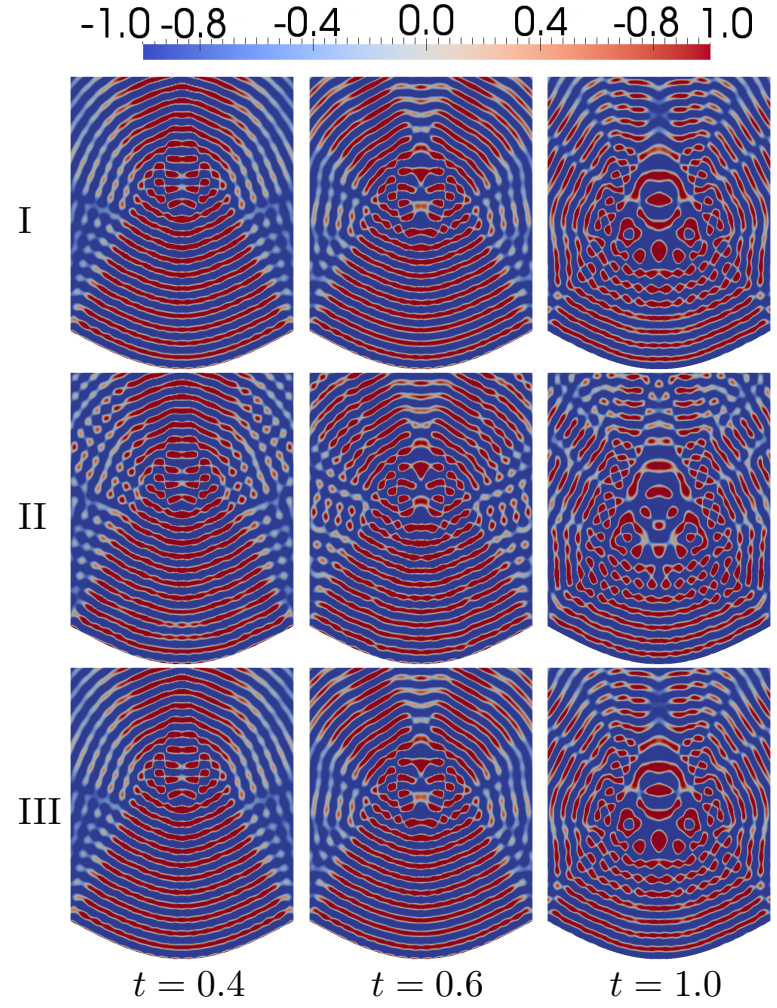

(a) Acoustic pressure

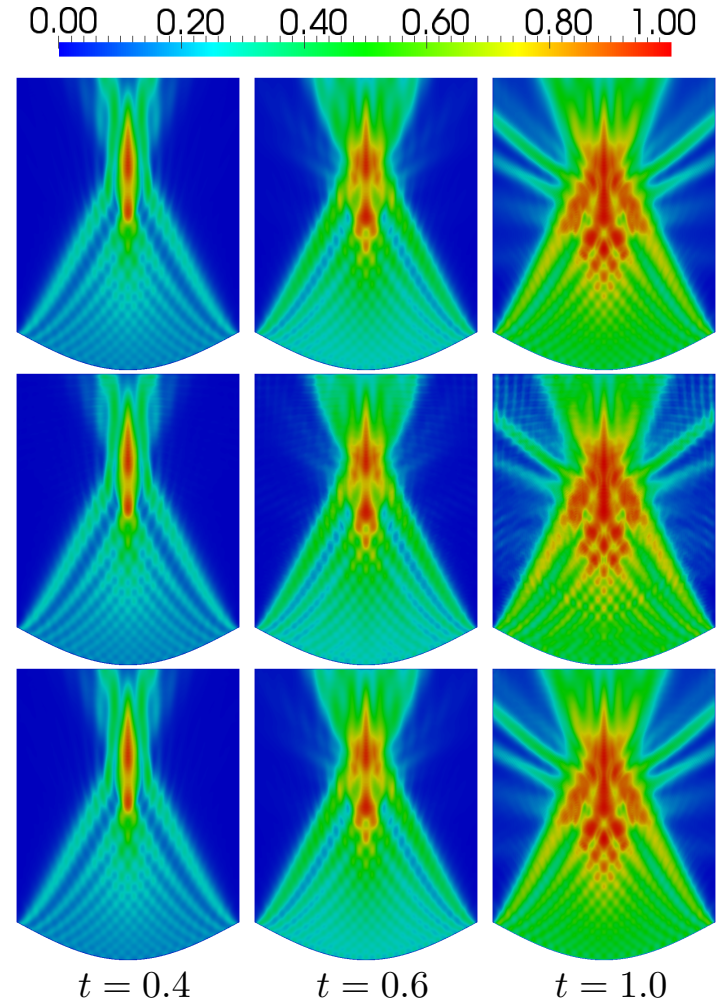

(b) Temperature field.

Fig. 7. A series of snapshots of the acoustic pressure and the temperature field for the excitation frequency $\omega=1.2 \mathrm{MHz}$ at different times. The reference solution is in row I. The first and second order ABCs (30) are in rows II and III, respectively.

We also analyze how the relative error of the acoustic pressure and the temperature propagates in time (see Fig. 8).

In the short term range both first and second order ABCs (30) are quite independent of the applied excitation frequency $\omega$. However, the situation is different in the long term range. Here, the first order ABC is very sensitive with respect to $\omega$. The higher the frequency is the higher the error is. This effect is drastically reduced by the use of the second order $\mathrm{ABC}$.

Another observation is that the first order $\mathrm{ABC}$ for different frequencies gives mostly the same accuracy for $t \leq 0.6$ (see Fig.8(a)) for any of the considered $\omega$ and becomes worse for $t>0.6$ as $\omega$ increases. This is explained by the thermo-acoustic lensing effect which manifests itself rather weakly up to $t \approx 0.6$. However, for $t>0.6$ its influence is more pronounced and makes the acoustic field more challenging for the first order ABC. In contrast, the second order ABC is of high accuracy in the short and long term range, and operates equally well for all studied frequencies. 


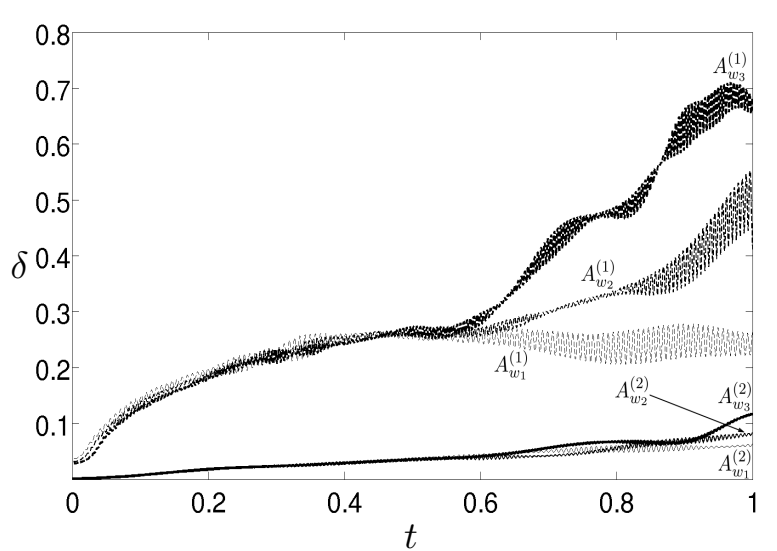

(a) Acoustic pressure.

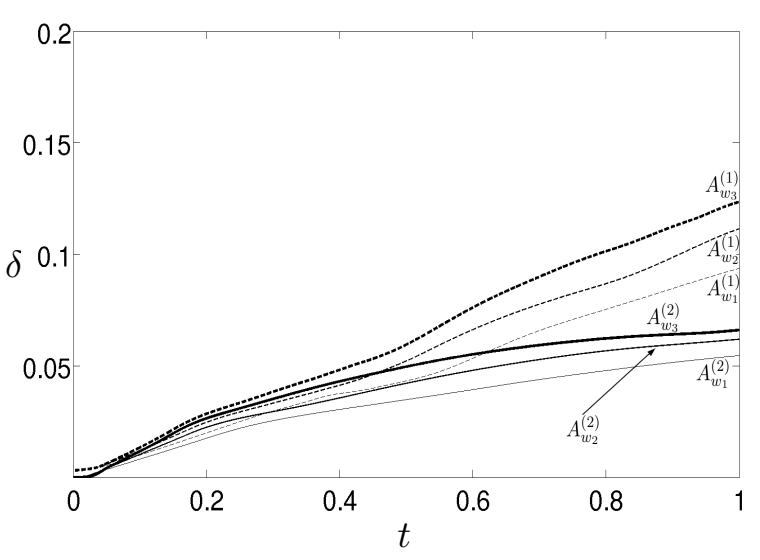

(b) Temperature field.

Fig. 8. The accuracy of the first $A^{(1)}$ and second $A^{(2)}$ order ABCs (30) for different excitation frequencies 61. Conchturions $\overline{\mathbf{s}} 1.0 \mathrm{MHz}, \omega_{3}=1.2 \mathrm{MHz}$. The relative error $\delta$ is given in the Euclidean norm.

In this paper, we propose new absorbing boundary conditions for the wave equation with a temperature dependent speed of sound. The well-posedness of the acoustic wave equation is shown and also confirmed by numerical simulations which exhibit no instabilities. All our experiments show that the first order $\mathrm{ABC}$ is computationally easier to handle than the second order one but it leads to a substantial loss of accuracy especially at high frequencies. The second order $\mathrm{ABC}$ is more accurate and provides quantitatively much better results in a wide range of excitation frequencies compared to the first order condition. To obtain a stable discrete formulation of the second order $\mathrm{ABC}$, we use a weak Lagrange multiplier formulation. Both proposed absorbing boundary conditions have low computational complexity due to their locality and can be implement into existing codes. We also would like to remark that the application of self-adapting $\mathrm{ABCS}^{55}$ to the thermo-acoustic problem will lead to a further improvement of the results.

\section{Acknowledgments}

The authors are grateful to the referees for valuable comments and suggestions. They also want to thank the German Research Foundation and the Austrian Science Foundation for the support of this work under grants WO 671/6-2 and I 533-N20.

\section{References}

1. A. Sommerfeld, Partial Differential Equations in Physics (Academic Press, New York, 1949).

2. B. Engquist, A. Majda, Absorbing boundary conditions for the numerical simulation of waves, Math. Comp. 31 (1977) 629.

3. A. Bayliss, E. Turkel, Radiation boundary conditions for wave-like equations, Comm. Pure Appl. Math. 33 (1980) 707.

4. A. Bayliss, C. Goldstein, E. Turkel, On accuracy conditions for the numerical computation of waves, J. Comput. Phys. 59 (1985) 396. 
5. B. Gustafsson, H.-O. Kreiss, Boundary conditions for time dependent problems with an artificial boundary, J. Comput. Phys. 30 (1979) 333.

6. T. Hagstrom, H. Keller, Exact boundary conditions at an artificial boundary for partial differential equations in cylinders, SIAM J. Math. Anal. 17 (1986) 322.

7. R. C. Maccamy, S. Marin, A finite element method for exterior interface problems, Int. J. Math. Math. Sci. 3 (1980) 311.

8. L. Ting, M. J. Miksis, Exact boundary conditions for scattering problems, J. Acoust. Soc. Am. 6 (1986) 1825.

9. J. Keller, D. Givoli, Exact non-reflecting boundary conditions, J. Comput. Phys. 82 (1989) 172.

10. V. Ryabenḱii, S. Tsynkov, Artificial boundary conditions for the numerical solution of external viscous flow problems, SIAM J. Numer. Anal. 32 (1995) 1355.

11. S. Tsynkov, S. Abarbanel, E. Turkel, External flow computations using global boundary conditions, AIAA Journal 34 (1996) 700.

12. T. Hagstrom, A. Mar-Or, D. Givoli, High-order local absorbing conditions for the wave equation: Extensions and improvements, J. Comput. Phys. 227 (2008) 3322.

13. R. Higdon, Radiation boundary conditions for dispersive waves, SIAM J. Numer. Anal. 31 (1994) 64.

14. A.-D. Cheng, D. Cheng, Heritage and early history of the boundary element method, Eng. Anal. Boundary Elem. 29 (2005) 268.

15. B. Nolte, I. Schäfer, J. Ehrlich, N. Ochmann, R. Burgschweiger, S. Marburg, Numerical methods for wave scattering phenomena by means of different boundary integral formulations, J. Comput. Acoust. 15 (2007) 495.

16. D. Burnett, A three-dimensional acoustic infinite element based on a prolate spheroidal multipole expansion, J. Acoust. Soc. Am. 96 (1994) 2798.

17. R. J. Astley, J. A. Hamilton, Numerical studies of conjugated infinite elements for acoustical radiation, J. Comput. Acoust. 8 (2000) 1.

18. D. Dreyer, O. von Estorff, Improved conditioning of infinite elements for exterior acoustics, Internat. J. Numer. Methods Engrg. 58 (2003) 933.

19. J. Baumgart, S. Marburg, S. Schneider, Efficient sound power computation of open strutures with infinite/finite elements and by means of Pade-via-Lanzos algorithm, J. Comput. Acoust. 15 (2007) 557.

20. J.-P. Berenger, A perfectly matched layer for the absorption of electromagnetic waves, J. Comput. Phys. 114 (1994) 185.

21. W. Chew, J. Jin, Perfectly matched layers in the discretized space: An analysis and optimization, Electromagnetics 16 (1996) 325.

22. E. Becache, S. Fauqueux, P. Joly, Stability of perfectly matched layers, group velocities, $J$. Comput. Phys. 188 (2003) 399.

23. F. Nataf, A new construction of perfectly matched layers for the linearized euler equations, $J$. Comput. Phys. 214 (2006) 757.

24. D. Appeloä, G. Kreiss, Application of a perfectly matched layer to the nonlinear wave equation, Wave Motion 44 (2007) 531.

25. S. Tsynkov, Numerical solution of problems on unbounded domains. A review, Appl. Numer. Math. 27 (1998) 465.

26. T. Hagstrom, Radiation boundary conditions for the numerical simulation of waves, Acta Numerica 8 (1999) 47.

27. D. Givoli, Computational absorbing boundaries, in Computational Acoustics of Noise Propagation in Fluids, eds. S. Marburg, B. Nolte (Springer-Verlag, Berlin Heidelberg, 2008), pp. 145-166.

28. B. Engquist, A. Majda, Radiation boundary conditions for acoustic and elastic wave calculations, Comm. Pure Appl. Math. 32 (1979) 313. 
29. G. W. Hedstrom, Nonreflecting boundary conditions for nonlinear hyperbolic systems, J. Comput. Phys. 30 (1979) 222.

30. E. Bécache, D. Givoli, T. Hagstrom, High-order absorbing boundary conditions for anisotropic and convective wave equations, J. Comput. Phys. 229 (2010) 1099.

31. J. Szeftel, Absorbing boundary conditions for nonlinear scalar partial differential equations, Comput. Method. Appl. M. 195 (2006) 3760.

32. J. Zhang, Z. Xu, X. Wu, Unified approach to split absorbing boundary conditions for nonlinear Schrödinger equations: Two-dimensional case, Phys. Rev. E 79 (2009) 046711-1.

33. R. R. Paz, M. A. Storti, L. Garelli, Absorbing boundary condition for nonlinear hyperbolic partial diferential equations with unknown Riemann invariants, Fluid Mechanics (C) XXVIII (2009) 1593.

34. S. Makarov, M. Ochmann, Nonlinear and thermoviscous phenomena in acoustics, part I, Acta Acustica united with Acustica 82 (1996) 579.

35. S. Makarov, M. Ochmann, Nonlinear and thermoviscous phenomena in acoustics, part II, Acta Acustica united with Acustica 83 (1997) 197.

36. K. Naugolnykh, L. Ostrovsky, Nonlinear Wave Processes in Acoustics (Cambridge University Press, London, 1998).

37. W. Nyborg, Heat generation by ultrasound in a relaxing medium, J. Acoust. Soc. Am. 70 (1981) 310.

38. J.-M. Bony, Calcul symbolique et propagation des singularités pour les équations aux dérivées non linéaires, Ann. Sci. École. Norm. Sup. 14 (1981) 209.

39. S. Benzoni-Gavage, D. Serre, Multidimensional hyperbolic partial differential equations. Firstorder systems and applications (Oxford University Press, 2007).

40. L. Nirenberg, Lectures on linear partial differential equations, Uspekhi Mat. Nauk 30 (1975) 147.

41. B. Engquist, A. Majda, Reflection of singularities at the boundary, Comm. Pure Appl. Math. XXVIII (1975) 479.

42. M. W. Wong, An introduction to pseudo-differential operators (World Scientific Publishing, Singapore, 1999).

43. B. Engquist, A. Majda, Numerical radiation boundary conditions for unsteady transsonic flow, J. Comput. Phys. 40 (1981) 91.

44. L. Wagatha, Approximation of pseudodifferential operators in absorbing boundary conditions for hyperbolic equations, Numer. Math. 42 (1983) 51.

45. J. Chung, G. Hulbert, A time integration algorithm for structural dynamics with improved numerical dissipation: The generalized $\alpha$-method, J. Appl. Mech. 60 (1993) 371.

46. C. W. Connor, K. Hynynen, Bio-acoustic thermal lensing and nonlinear propagation in focused ultrasound surgery using large focal spots: a parametric study, Phys. Med. Biol. 47 (2002) 1911.

47. I. Hallaj, R. Cleveland, K. Hynynen, Simulations of the thermo-acoustic lens effect during focused ultrasound surgery, J. Acoust. Soc. Am., 109 (2001) 2245.

48. C. Le Floch, M. Tanter, M. Fink, Self-defocusing in ultrasonic hyperthermia: Experiment and simulation, Appl. Phys. Lett., 74 (1999) 3062.

49. C. Le Floch, M. Fink, Ultrasonic mapping of temperature in hyperthermia: the thermal lens effect, Proceedings of 1997 IEEE Ultrasonics Symposium, (1997) 1301.

50. C. Simon, P. VanBaren, E.S. Ebbini, Two-dimensional temperature estimation using diagnostic ultrasound, IEEE Trans. Ultrason. Ferr., 45 (1998) 1088.

51. M. Pernot, K.R. Waters, J. Bercoff, M. Tanter, M. Fink, Reduction of the thermo-acoustic lens effect during ultrasound-based temperature estimation, Proceedings of 2002 IEEE Ultrasonics Symposium, (2002) 1447.

52. A. Bambergern, P. Joly, J. Roberts, Second-Order Absorbing Boundary Conditions for the Wave 
July 13, 2012 16:24 WSPC/130-JCA OnABCforWaveEquWithTemperatureDependentC ${ }^{*} 11$

20 I. Shevchenko, M. Kaltenbacher, B. Wohlmuth

Equation: A Solution for the Corner Problem, SIAM J. Numer. Anal., 27 (1990) 323.

53. T. Ha-Duong, P. Joly, On the Stability Analysis of Boundary Conditions for the Wave Equation by Energy Methods. Part I: The Homogeneous Case, Math. Comp., 62 (1994) 539.

54. V. Gordienko, Symmetrization of mixed problem for second-order hyperbolic equation with two space variables, Sib. Mat. Zh., 22 (1981) 84.

55. I. Shevchenko, B. Wohlmuth, Self-adapting absorbing boundary conditions for the wave equation, Wave Motion, 49 (2012) 461.

56. I. Shevchenko, M. Kaltenbacher, B. Wohlmuth, A multi-time stepping integration method for the ultrasound heating problem, Accepted for publication in ZAMM, (2012).

57. A. Knapp, Advanced real analysis (Birkhäuser, USA, 2005).

58. T. Rossing(Ed.), Handbook of Acoustics (Springer, 2007).

59. H. Kuttruff, Acoustics. An introduction (Taylor \& Francis, New York, 2007).

60. T. Hughes, The finite element method: linear static and dynamic finite element analysis (Prentice-Hall, New Jersey, 1987). 